\title{
19. PRELIMINARY PALEOMAGNETIC MEASUREMENTS ON SEDIMENTS FROM THE IONIAN (SITE 125) AND TYRRHENIAN (SITE 132) BASINS OF THE MEDITERRANEAN SEA
}

\author{
William B. F. Ryan and Jane D. Flood, Lamont-Doherty Geological Observatory \\ of Columbia University, Palisades, New York
}

\section{INTRODUCTION}

The intensity and direction of remanent magnetization have been measured in oriented sediment samples from Site 125 in the Ionian Basin and Site 132 in the Tyrrhenian Basin. These preliminary measurements were undertaken in an effort to determine if the techniques of paleomagnetism could reveal a chronology of magnetic field reversals in continuously cored Pleistocene and Pliocene sequences.

For some time, debate has focused on whether biostratigraphic levels established in open marine sequences are time-synchronous with other levels described in the "typesections" recognized by the Subcommission for Stratigraphic Classification and Terminology. It was anticipated that deep-sea sequences of pelagic ooze at locations relatively close to the Italian stratotypes for the Neogene might provide a correlation link between the Mediterranean biozonations and those established outside the area, if an identifiable reversal sequence could be found in the Mediterranean drill cores.

As will be elaborated in Chapter 47.2, this initial attempt has been most disappointing, primarily because the magnetic data have been very difficult to decipher in an unambiguous fashion. However, some limited conclusions can be reached, and thus all of the data are presented and discussed.

\section{PROCEDURE FOR THE SAMPLING AND MEASUREMENT OF MAGNETIC PROPERTIES}

The DSDP cores are unoriented in terms of azimuth when cut from the seabed and retrieved. However, under the assumption that the drill string enters the sediment layers in the near-vertical direction, it remains a rather simple procedure to preserve the up and down direction of each nine-meter core barrel. Since the latitudes of the Mediterranean sites are sufficiently far north of the magnetic equator, the vertical component of magnetic field (inclination) is theoretically steep enough to record whether the sediment material is normally or reversely magnetized.

The up and down direction of the split cores was noted when removing an oriented cylinder $(2.4 \mathrm{~cm}$ long $X 2.5 \mathrm{~cm}$ diameter) of sediment with non-magnetic devices. Precautions against improper orientation were taken by sampling only at levels in the cores where easily recognizable lithologic boundaries (original bedding surfaces) were horizontal across the split face of the cores. Furthermore, no samples were cut from highly plastic or watery sections where the possibility existed that sediment grains may have had a chance to re-orient themselves during the coring recovery, or storage procedures.

\section{Spinning}

The still moist samples were initially measured on a $5 \mathrm{~Hz}$ spinner magnetometer (Foster, 1966) without A-C demagnetization (cleaning). This was followed by a determination of their weak field susceptibilities. Ideally, a progressive demagnetization curve should be obtained for each individual specimen. However, because of the large number of samples, it seemed more expedient to choose a set of representative specimens after analysis of the NRM measurements, and investigate their magnetic stabilities first.

Examination of the demagnetization curves (Figure 1) reveals a significant intensity decrease followed by a gradual leveling of the curve in only three of the samples. It appeared that, for these sediments, treatment of 100 oersted is sufficient to remove most of the spurious magnetic components.

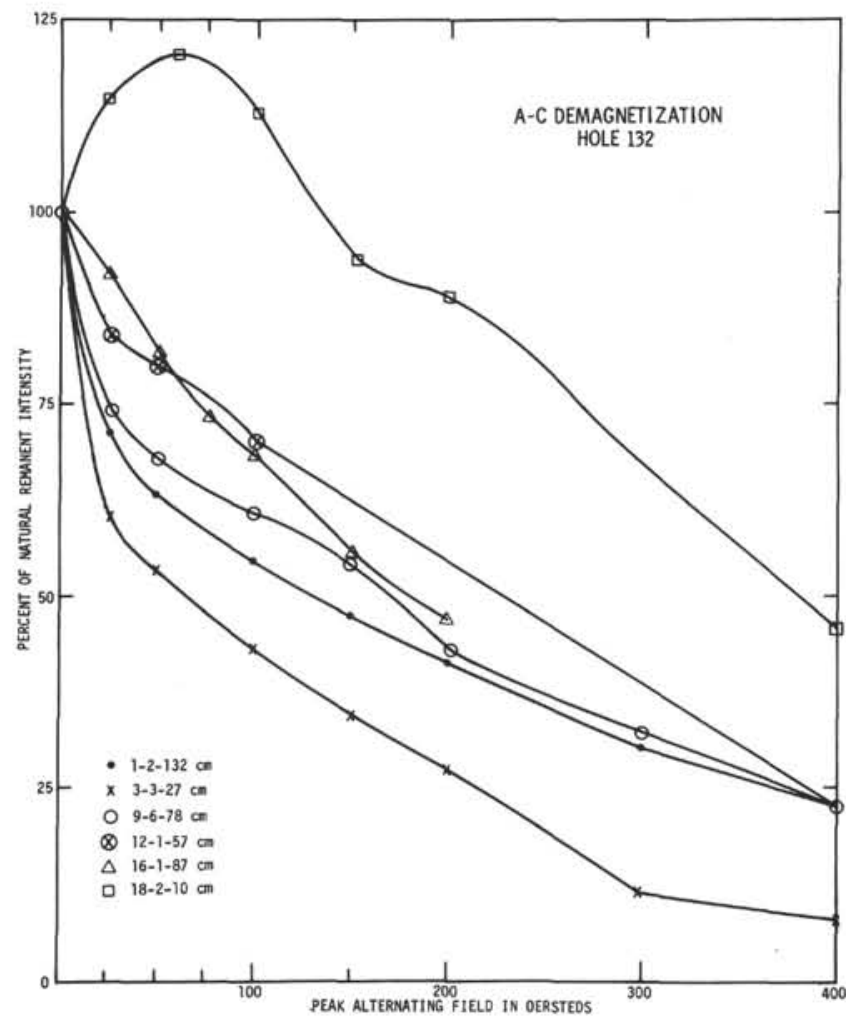

Figure 1. Demagnetization curves for selected samples from Hole 132. 
One of the samples actually showed an increase in intensity upon cleaning until a field of 150 oersteds was reached, and the other two showed a uniform decrease suggesting that treatment up to 400 oersted was still insufficient to remove secondary magnetic components.

Because of the demagnetization behavior of the selected specimens, all the remaining samples were partially demagnetized in a $60 \mathrm{~Hz}$ alternating field of 100 oersted in an apparatus similar to that described by McElhinny (1966).

\section{RESULTS}

The directions of inclination of the natural remanent magnetization (NRM), the inclinations after treatment, the NRM intensities, and specific susceptibilities (normalized by weighing of room-temperature dried samples) are listed in Tables 1 and 2, and are shown graphically in Figures 2 and 3.

Inspection of the figures shows that inclination values vary considerably and have a preponderance of normal polarities $(+$ is downward in the northern hemisphere and indicates the sign of the present normal field). The intensities range from $10^{-4}$ to $10^{-6} \mathrm{emu} / \mathrm{gm}$, where $10^{-4}$ is considered a reasonably strong value for carbonate rich marine sediments. Furthermore, the general demagnetization treatment at 100 oersteds did not seem to alter significantly the inclinations or intensities, particularly at Sites 125 and 125A. Some of the specimens that had negative NRM inclinations changed to positive values after washing, whereas others changed to even higher negative values. Very few of the negative inclinations, even after washing at 100 oersteds, approach a value consistent with the vector direction of a geocentric dipole field for the given latitude of the site.

Of particular interest is the wide range in susceptibilities and the rather systematic variations in the susceptibility curves. Since this is an inherent property of the sediment resulting from its proportional makeup of magnetic minerals, the levels of high and low susceptibility in the sediment column may reflect variations in sediment source and/or grain size and consequently may be useful parameters in cross-correlating strata to other nearby locations. (See discussion of the correlation of Sites 132 and 125 in Chapter 47).

\section{DISCUSSION}

The interpretation of the paleomagnetic inclination measurements in terms of the established paleomagnetic reversal time scale (Cox et al., 1963; Dalrymple et al., 1967; Foster and Opdyke, 1970) is depicted in Figures 2 and 3. A full discussion of this interpretation is reserved for Chapter 47.2 (this volume) on the paleomagnetic stratigraphy of the Mediterranean cores. In that chapter, the magnetic reversal sequence is correlated to biostratigraphic horizons and established datum levels. The quality of the measurements and the reliability of the inferred stratigraphy is also evaluated.

\section{ACKNOWLEDGMENTS}

We wish to acknowledge the kind cooperation provided by R. J. Fiske during the sampling of the DSDP cores at the east coast repository. The analytical measurements and procedures for $\mathrm{A}-\mathrm{C}$ demagnetization were carried out under the supervision of William Lowrie and Neil D. Opdyke. Discussions with Dennis Kent have been very helpful. Lamont-Doherty Geological Observatory Contribution No. 1875 .

TABLE 1

Magnetic Measurements of Selected Samples from Holes 125 and 125A

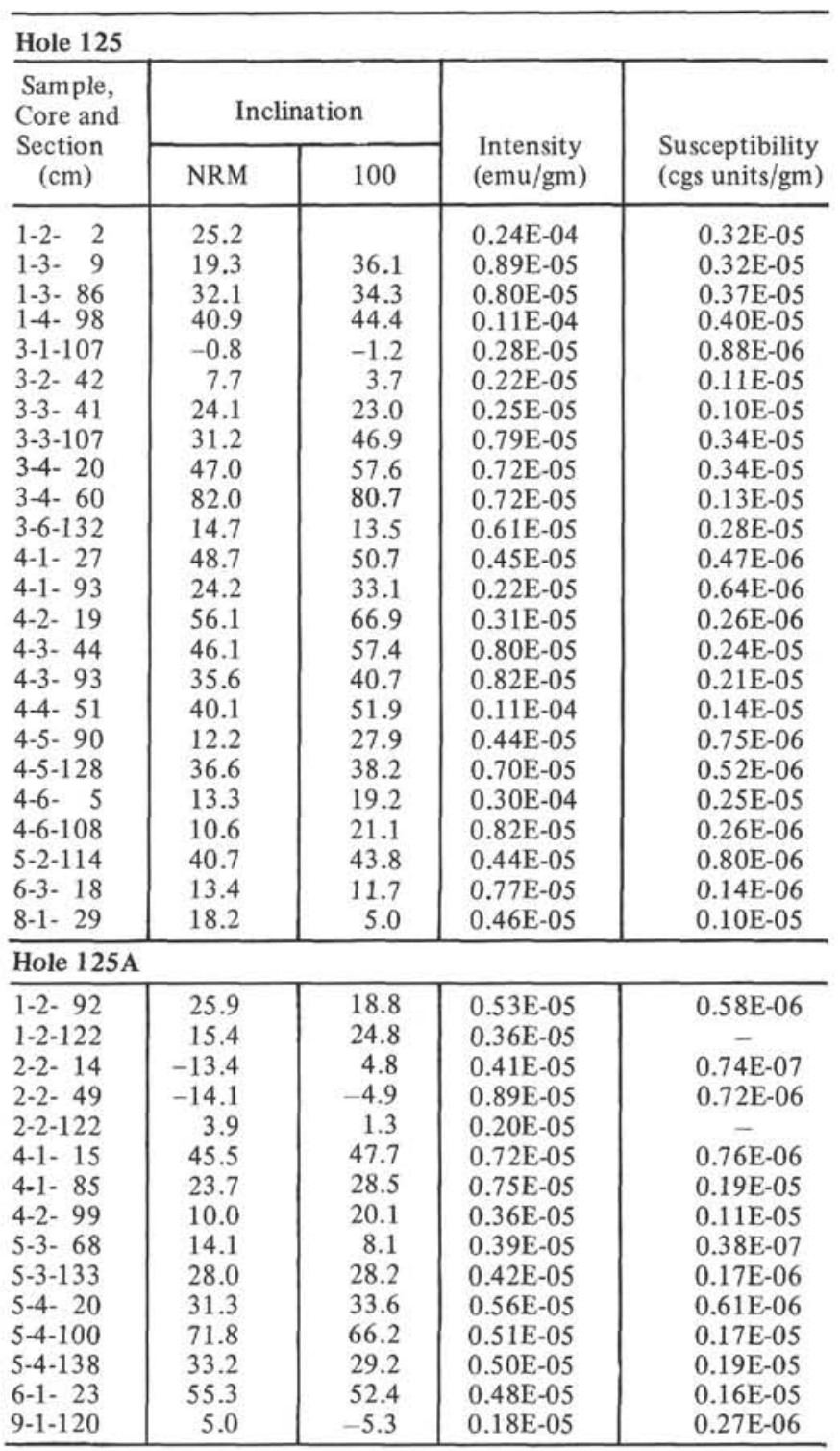


TABLE 2

Magnetic Measurements of Selected Samples from Hole 132

\begin{tabular}{|c|c|c|c|c|}
\hline \multicolumn{5}{|l|}{ Hole 132} \\
\hline \multirow{2}{*}{$\begin{array}{l}\text { Sample, } \\
\text { Core and } \\
\text { Section } \\
(\mathrm{cm})\end{array}$} & \multicolumn{2}{|c|}{ Inclination } & \multirow{2}{*}{$\begin{array}{l}\text { Intensity } \\
\text { (emu/gm) }\end{array}$} & \multirow{2}{*}{$\begin{array}{l}\text { Susceptibility } \\
\text { (cgs units/gm) }\end{array}$} \\
\hline & NRM & 100 & & \\
\hline $1-1-20$ & 20.6 & 17.0 & $0.92 \mathrm{E}-05$ & $0.88 \mathrm{E}-05$ \\
\hline $1-1-70$ & 15.3 & 18.0 & $0.77 \mathrm{E}-05$ & $0.73 \mathrm{E}-05$ \\
\hline $1-2-31$ & 50.7 & 52.8 & $0.22 \mathrm{E}-04$ & $0.56 \mathrm{E}-05$ \\
\hline $1-2-132$ & 56.2 & 55.8 & $0.15 \mathrm{E}-04$ & $0.74 \mathrm{E}-05$ \\
\hline $1-5-67$ & 27.6 & - & $0.53 \mathrm{E}-05$ & $0.58 \mathrm{E}-05$ \\
\hline $1-6-134$ & 71.9 & 64.9 & $0.48 \mathrm{E}-05$ & $0.86 \mathrm{E}-05$ \\
\hline $2-3-86$ & 67.3 & 64.8 & $0.20 \mathrm{E}-04$ & $0.85 \mathrm{E}-05$ \\
\hline $2-4-\quad 8$ & -80.0 & 82.3 & $0.97 \mathrm{E}-05$ & $0.82 \mathrm{E}-05$ \\
\hline $2-4-115$ & -43.1 & 66.6 & $0.96 \mathrm{E}-05$ & $0.17 \mathrm{E}-05$ \\
\hline $3-3-27$ & -30.6 & 54.9 & $0.10 \mathrm{E}-04$ & $0.77 \mathrm{E}-05$ \\
\hline $3-4-91$ & -26.9 & -11.1 & $0.19 \mathrm{E}-04$ & $0.79 \mathrm{E}-05$ \\
\hline $4-3-55$ & 11.5 & -5.6 & $0.61 \mathrm{E}-04$ & $0.59 \mathrm{E}-05$ \\
\hline $4-3-131$ & 74.9 & 66.9 & $0.41 \mathrm{E}-05$ & $0.55 \mathrm{E}-05$ \\
\hline $4-4-26$ & -11.3 & 28.2 & $0.25 \mathrm{E}-05$ & $0.61 \mathrm{E}-05$ \\
\hline $4-5-42$ & 22.8 & 58.2 & $0.15 \mathrm{E}-04$ & $0.31 \mathrm{E}-06$ \\
\hline $4-6-65$ & 29.7 & 52.1 & $0.14 \mathrm{E}-04$ & $0.72 \mathrm{E}-06$ \\
\hline $4-6-142$ & 53.0 & 84.6 & $0.26 \mathrm{E}-04$ & $0.45 \mathrm{E}-06$ \\
\hline $6-3-34$ & 73.5 & 47.4 & $0.14 \mathrm{E}-04$ & $0.12 \mathrm{E}-05$ \\
\hline $6-3-130$ & 70.4 & 19.8 & $0.12 \mathrm{E}-04$ & $0.60 \mathrm{E}-05$ \\
\hline $6-4-\quad 4$ & -20.1 & 71.6 & $0.77 \mathrm{E}-05$ & $0.30 \mathrm{E}-05$ \\
\hline $6-5-32$ & 69.8 & 77.0 & $0.21 \mathrm{E}-04$ & $0.27 \mathrm{E}-05$ \\
\hline $6-6-10$ & 60.7 & 75.4 & $0.22 \mathrm{E}-04$ & $0.83 \mathrm{E}-06$ \\
\hline $7-1-63$ & 57.6 & 83.5 & $0.25 \mathrm{E}-04$ & $0.11 \mathrm{E}-05$ \\
\hline $7-3-118$ & 28.0 & 69.5 & $0.12 \mathrm{E}-04$ & - \\
\hline $7-4-44$ & -12.4 & 75.8 & $0.94 \mathrm{E}-05$ & $0.19 \mathrm{E}-05$ \\
\hline $7-5-76$ & -6.2 & 65.5 & $0.12 \mathrm{E}-04$ & $0.13 \mathrm{E}-05$ \\
\hline $7-6-18$ & 62.8 & 68.1 & $0.55 \mathrm{E}-05$ & $0.11 \mathrm{E}-05$ \\
\hline $7-6-56$ & 83.8 & 82.4 & $0.55 \mathrm{E}-05$ & $0.18 \mathrm{E}-05$ \\
\hline $8-4-34$ & 68.7 & 55.5 & $0.95 \mathrm{E}-05$ & $0.25 \mathrm{E}-05$ \\
\hline $8-4-74$ & 70.6 & 65.1 & $0.25 \mathrm{E}-04$ & $0.30 \mathrm{E}-05$ \\
\hline $8-5-89$ & 75.3 & 49.2 & $0.94 \mathrm{E}-05$ & $0.33 \mathrm{E}-05$ \\
\hline $8-6-9$ & 73.4 & 65.2 & $0.42 \mathrm{E}-05$ & $0.29 \mathrm{E}-05$ \\
\hline $8-6-45$ & 32.9 & 67.3 & $0.14 \mathrm{E}-04$ & $0.19 \mathrm{E}-05$ \\
\hline $9-3-30$ & 75.9 & 66.8 & $0.28 \mathrm{E}-05$ & $0.41 \mathrm{E}-06$ \\
\hline $9-4-48$ & 79.4 & 79.1 & $0.77 \mathrm{E}-05$ & $0.34 \mathrm{E}-05$ \\
\hline $9-4-147$ & 64.0 & 66.9 & $0.10 \mathrm{E}-04$ & $0.52 \mathrm{E}-05$ \\
\hline $9-5-45$ & 84.1 & - & $0.43 \mathrm{E}-05$ & $0.20 \mathrm{E}-05$ \\
\hline $9-5-135$ & 55.8 & 56.9 & $0.11 \mathrm{E}-04$ & - \\
\hline $9-6-78$ & -8.0 & 71.0 & $0.83 \mathrm{E}-05$ & $0.52 \mathrm{E}-06$ \\
\hline $9-6-137$ & 64.9 & 71.4 & $0.22 \mathrm{E}-04$ & $0.52 \mathrm{E}-06$ \\
\hline $10-3-20$ & 47.2 & 49.1 & $0.14 \mathrm{E}-04$ & $0.37 \mathrm{E}-06$ \\
\hline $10-3-137$ & 59.0 & 75.8 & $0.16 \mathrm{E}-04$ & $0.13 \mathrm{E}-05$ \\
\hline $10-4-16$ & 73.2 & 75.3 & $0.13 \mathrm{E}-04$ & $0.14 \mathrm{E}-05$ \\
\hline $10-4-129$ & 29.3 & 78.2 & $0.13 \mathrm{E}-04$ & $0.17 \mathrm{E}-05$ \\
\hline $10-5-71$ & 31.9 & 58.8 & $0.18 \mathrm{E}-04$ & $0.17 \mathrm{E}-05$ \\
\hline $10-5-113$ & 61.6 & 58.2 & $0.16 \mathrm{E}-04$ & $0.44 \mathrm{E}-05$ \\
\hline $10-6-73$ & 70.8 & 59.5 & $0.83 \mathrm{E}-05$ & $0.15 \mathrm{E}-05$ \\
\hline $10-6-117$ & 81.6 & 58.6 & $0.69 \mathrm{E}-05$ & $0.17 \mathrm{E}-05$ \\
\hline $11-2-21$ & 1.7 & -18.1 & $0.94 \mathrm{E}-05$ & $0.43 \mathrm{E}-05$ \\
\hline $11-3-65$ & 84.0 & 82.9 & $0.13 \mathrm{E}-04$ & $0.33 \mathrm{E}-05$ \\
\hline $11-4-50$ & 73.0 & 75.1 & $0.95 \mathrm{E}-05$ & $0.31 \mathrm{E}-05$ \\
\hline $11-5-49$ & 80.0 & 71.6 & $0.12 \mathrm{E}-04$ & $0.34 \mathrm{E}-05$ \\
\hline $11-6-14$ & 81.0 & 58.9 & $0.32 \mathrm{E}-05$ & $0.30 \mathrm{E}-05$ \\
\hline $11-6-89$ & 69.8 & 56.3 & $0.46 \mathrm{E}-05$ & $0.69 \mathrm{E}-07$ \\
\hline $12-1-57$ & 68.6 & 64.9 & $0.11 \mathrm{E}-04$ & $0.14 \mathrm{E}-06$ \\
\hline $12-2-\quad 4$ & 69.1 & 55.1 & $0.52 \mathrm{E}-05$ & $0.17 \mathrm{E}-06$ \\
\hline $12-4-14$ & 73.8 & 47.6 & $0.50 \mathrm{E}-05$ & - \\
\hline $12-5-67$ & 65.5 & 36.4 & $0.46 \mathrm{E}-05$ & $0.30 \mathrm{E}-05$ \\
\hline $12-6-14$ & -3.5 & -8.5 & $0.47 \mathrm{E}-04$ & $0.69 \mathrm{E}-06$ \\
\hline $12-6-79$ & -13.0 & -10.2 & $0.96 \mathrm{E}-06$ & $0.68 \mathrm{E}-06$ \\
\hline $13-1-40$ & 74.1 & 67.9 & $0.61 \mathrm{E}-05$ & $0.18 \mathrm{E}-05$ \\
\hline $13-2-59$ & 69.8 & 67.2 & $0.83 \mathrm{E}-05$ & $0.38 \mathrm{E}-05$ \\
\hline $13-3-16$ & 76.2 & 71.8 & $0.75 \mathrm{E}-05$ & $0.36 \mathrm{E}-05$ \\
\hline $13-4-102$ & 85.7 & 69.5 & $0.76 \mathrm{E}-05$ & $0.34 \mathrm{E}-05$ \\
\hline $13-5-73$ & 70.7 & 56.7 & $0.52 \mathrm{E}-05$ & $0.24 \mathrm{E}-05$ \\
\hline $13-6-100$ & -57.6 & -59.7 & $0.23 \mathrm{E}-05$ & $0.14 \mathrm{E}-05$ \\
\hline
\end{tabular}

TABLE 2 - Continued

\begin{tabular}{|c|c|c|c|c|}
\hline \multirow{2}{*}{$\begin{array}{l}\text { Sample, } \\
\text { Core and } \\
\text { Section } \\
(\mathrm{cm})\end{array}$} & \multicolumn{2}{|c|}{ Inclination } & \multirow{2}{*}{$\begin{array}{l}\text { Intensity } \\
\text { (emu/gm) }\end{array}$} & \multirow{2}{*}{$\begin{array}{l}\text { Susceptibility } \\
\text { (cgs units/gm) }\end{array}$} \\
\hline & NRM & 100 & & \\
\hline $14-2-66$ & 63.9 & 50.8 & $0.19 \mathrm{E}-05$ & $0.72 \mathrm{E}-06$ \\
\hline $14-3-138$ & -32.6 & -10.6 & $0.31 \mathrm{E}-05$ & $0.62 \mathrm{E}-06$ \\
\hline $15-1-58$ & 54.8 & 37.6 & $0.25 \mathrm{E}-05$ & $0.17 \mathrm{E}-06$ \\
\hline $15-2-98$ & 80.0 & 59.8 & $0.29 \mathrm{E}-05$ & $0.20 \mathrm{E}-06$ \\
\hline $15-3-148$ & 69.2 & 37.3 & $0.23 \mathrm{E}-05$ & $0.37 \mathrm{E}=06$ \\
\hline $15-4-11$ & 46.2 & 50.2 & $0.20 \mathrm{E}-05$ & $0.17 \mathrm{E}-06$ \\
\hline $15-4 \quad 49$ & 85.1 & 47.9 & $0.25 \mathrm{E}-05$ & $0.26 \mathrm{E}-06$ \\
\hline $15-5-6$ & 67.7 & 42.7 & $0.36 \mathrm{E}-05$ & $0.33 \mathrm{E}-06$ \\
\hline $15-5-133$ & 66.2 & 39.6 & $0.24 \mathrm{E}-05$ & $0.15 \mathrm{E}-06$ \\
\hline $15-6-33$ & 66.0 & 21.5 & $0.20 \mathrm{E}-05$ & $0.45 \mathrm{E}-06$ \\
\hline $15-6-138$ & 15.0 & -1.8 & $0.62 \mathrm{E}-05$ & $0.79 \mathrm{E}-06$ \\
\hline $16-1-87$ & 64.4 & 68.6 & $0.74 \mathrm{E}-05$ & $0.65 \mathrm{E}-06$ \\
\hline $16-2-49$ & 70.4 & 70.1 & $0.47 \mathrm{E}-05$ & $0.52 \mathrm{E}-06$ \\
\hline $16-3-4$ & 89.1 & 69.9 & $0.60 \mathrm{E}-05$ & $0.13 \mathrm{E}-06$ \\
\hline $16-3-58$ & 87.3 & 74.5 & $0.79 \mathrm{E}-05$ & $0.26 \mathrm{E}-06$ \\
\hline $16-4-\quad 2$ & 80.4 & 70.9 & $0.69 \mathrm{E}-05$ & $0.11 \mathrm{E}-05$ \\
\hline $16-5-22$ & 72.3 & 57.8 & $0.55 \mathrm{E}-05$ & $0.51 \mathrm{E}-06$ \\
\hline $16-5-63$ & 72.2 & 81.0 & $0.49 \mathrm{E}-05$ & $0.73 \mathrm{E}-06$ \\
\hline $16-6-32$ & 56.1 & 41.1 & $0.30 \mathrm{E}-05$ & $0.65 \mathrm{E}-06$ \\
\hline $16-6-95$ & 64.1 & 31.2 & $0.13 \mathrm{E}-05$ & $0.98 \mathrm{E}-06$ \\
\hline $17-2-134$ & 74.1 & 59.8 & $0.90 \mathrm{E}-05$ & $0.21 \mathrm{E}-05$ \\
\hline $17-3-10$ & 65.1 & 53.6 & $0.56 \mathrm{E}-05$ & $0.11 \mathrm{E}-05$ \\
\hline $17-4-42$ & 55.8 & 39.0 & $0.51 \mathrm{E}-05$ & $0.93 \mathrm{E}-06$ \\
\hline $17-4-142$ & 72.5 & 53.2 & $0.54 \mathrm{E}-05$ & $0.35 \mathrm{E}-06$ \\
\hline $17-5-19$ & 62.6 & 56.3 & $0.75 \mathrm{E}-05$ & $0.20 \mathrm{E}-05$ \\
\hline $17-5-95$ & 58.9 & 53.0 & $0.12 \mathrm{E}-05$ & $0.97 \mathrm{E}-06$ \\
\hline $17-6-69$ & 66.3 & 28.0 & $0.28 \mathrm{E}-05$ & $0.14 \mathrm{E}-05$ \\
\hline $18-1-78$ & 67.7 & 55.3 & $0.40 \mathrm{E}-05$ & $0.71 \mathrm{E}-06$ \\
\hline $18-2-10$ & -44.4 & -60.7 & $0.17 \mathrm{E}-05$ & $0.10 \mathrm{E}-05$ \\
\hline $18-2-112$ & 60.1 & 42.1 & $0.34 \mathrm{E}-05$ & $0.14 \mathrm{E}-05$ \\
\hline $18-3-40$ & 48.0 & 24.1 & $0.24 \mathrm{E}-05$ & $0.20 \mathrm{E}-05$ \\
\hline $18-4-30$ & 37.6 & 6.7 & $0.38 \mathrm{E}-05$ & $0.73 \mathrm{E}-06$ \\
\hline $18-4-136$ & 40.6 & 10.1 & $0.16 \mathrm{E}-05$ & $0.10 \mathrm{E}-05$ \\
\hline $18-5-22$ & 77.8 & 51.5 & $0.33 \mathrm{E}-05$ & $0.21 \mathrm{E}-05$ \\
\hline $18-5-134$ & 7.5 & 28.6 & $0.11 \mathrm{E}-05$ & $0.16 \mathrm{E}-05$ \\
\hline $18-6-58$ & 44.5 & 31.2 & $0.22 \mathrm{E}-05$ & $0.15 \mathrm{E}-05$ \\
\hline $19-2-88$ & 67.0 & 48.6 & $0.31 \mathrm{E}-05$ & $0.10 \mathrm{E}-05$ \\
\hline $19-3-84$ & 82.5 & 70.0 & $0.26 \mathrm{E}-05$ & $0.12 \mathrm{E}-05$ \\
\hline $19-4-7$ & 57.1 & 62.1 & $0.34 \mathrm{E}-05$ & $0.24 \mathrm{E}-05$ \\
\hline $19-4-127$ & 69.8 & 45.9 & $0.43 \mathrm{E}-05$ & - \\
\hline $20-2-8$ & 38.5 & 45.4 & $0.80 \mathrm{E}-05$ & - \\
\hline $20-2-82$ & 40.8 & 40.9 & $0.40 \mathrm{E}-05$ & - \\
\hline $20-3-87$ & 36.7 & 28.1 & $0.28 \mathrm{E}-05$ & $0.21 \mathrm{E}-05$ \\
\hline $20-4-24$ & 39.0 & 31.3 & $0.20 \mathrm{E}-05$ & $0.38 \mathrm{E}-05$ \\
\hline $20-4-84$ & 25.6 & 39.9 & $0.51 \mathrm{E}-05$ & $0.35 \mathrm{E}-05$ \\
\hline $21-1-106$ & 71.7 & 28.6 & $0.14 \mathrm{E}-04$ & $0.61 \mathrm{E}-05$ \\
\hline
\end{tabular}

\section{REFERENCES}

Cox, A., Doell, R. R. and Dalrymple, G. B., 1963. Geomagnetic polarity epochs. Science, 142, 382 .

Dalrymple, G. B., Cox, A., Doell, R. R. and Gromme, C. S., 1967. Pliocene geometric polarity epochs. Earth Planet. Sci. Lett., 2, 163.

Foster, J. H., 1966. A paleomagnetic spinner magnetometer using a fluxgate gradiometer. Earth Planet. Sci. Lett., 1, 463.

Foster, J. H. and Opdyke, N. D., 1970. Upper Miocene to Recent magnetic stratigraphy in deep-sea sediments. Jour. Geophys. Res., 75, 4465.

McElhinny, M. W., 1966. An improved method for demagnetizing rocks in alternating fields. Geophys. J. Roy. Astron. Soc., 10, 369. 


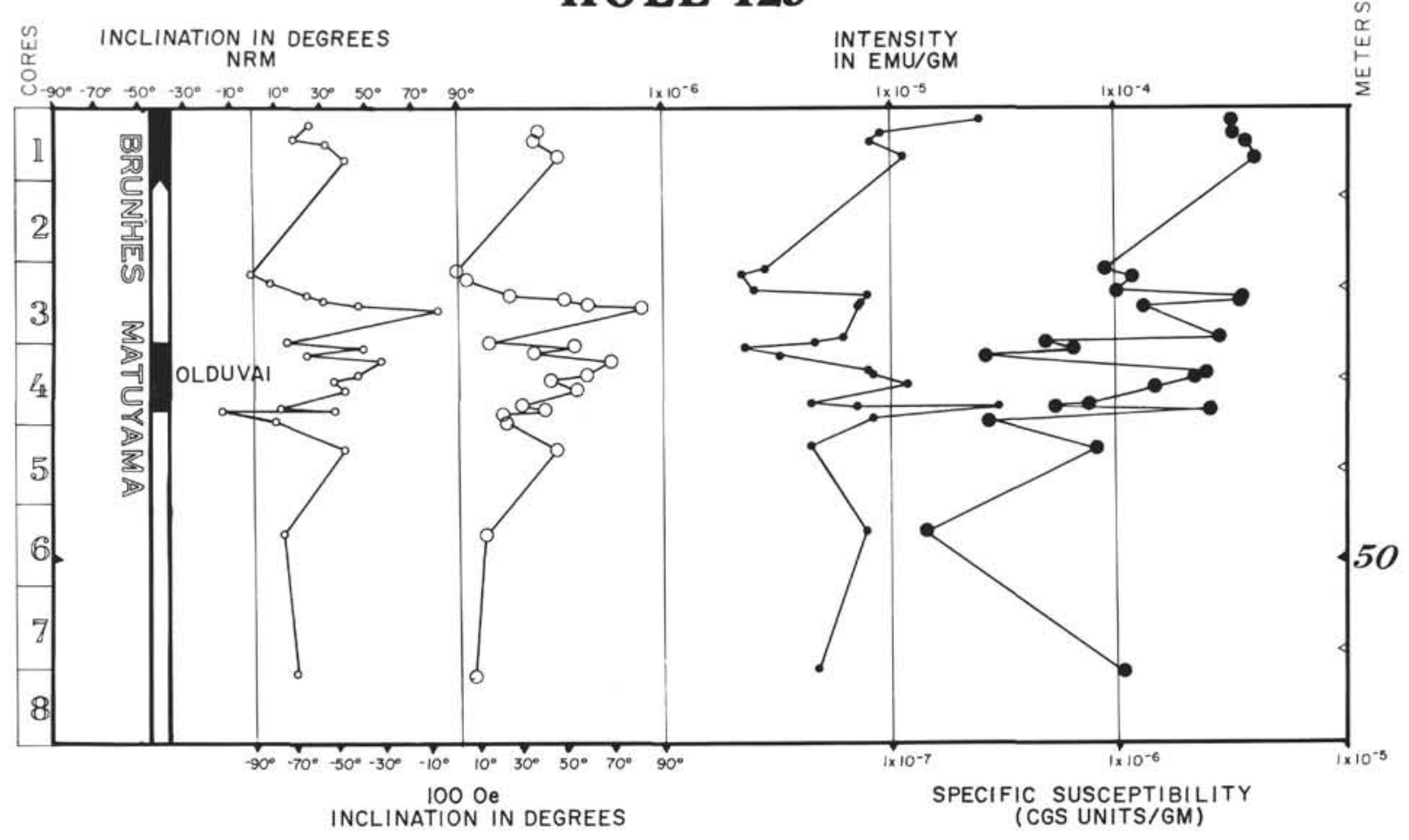

HOLE 125A

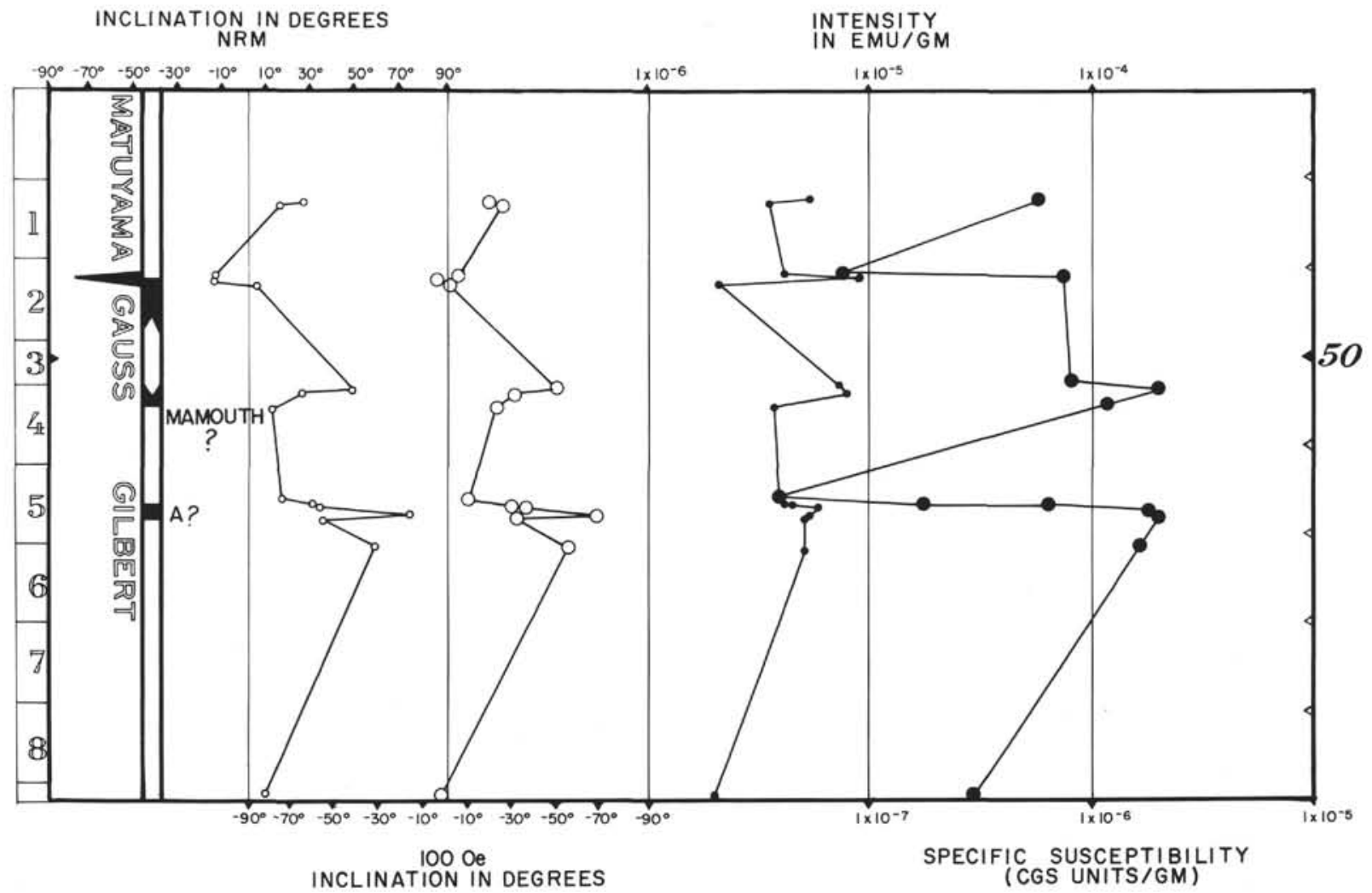

Figure 2. Inclination, intensity, and susceptibility profiles for Holes 125 and $125 \mathrm{~A}$. The inclination values are both before demagnetization (NRM) and after cleaning in a field of 100 oersted. The solid bars represent the preliminary interpretation of normal polarity periods. 


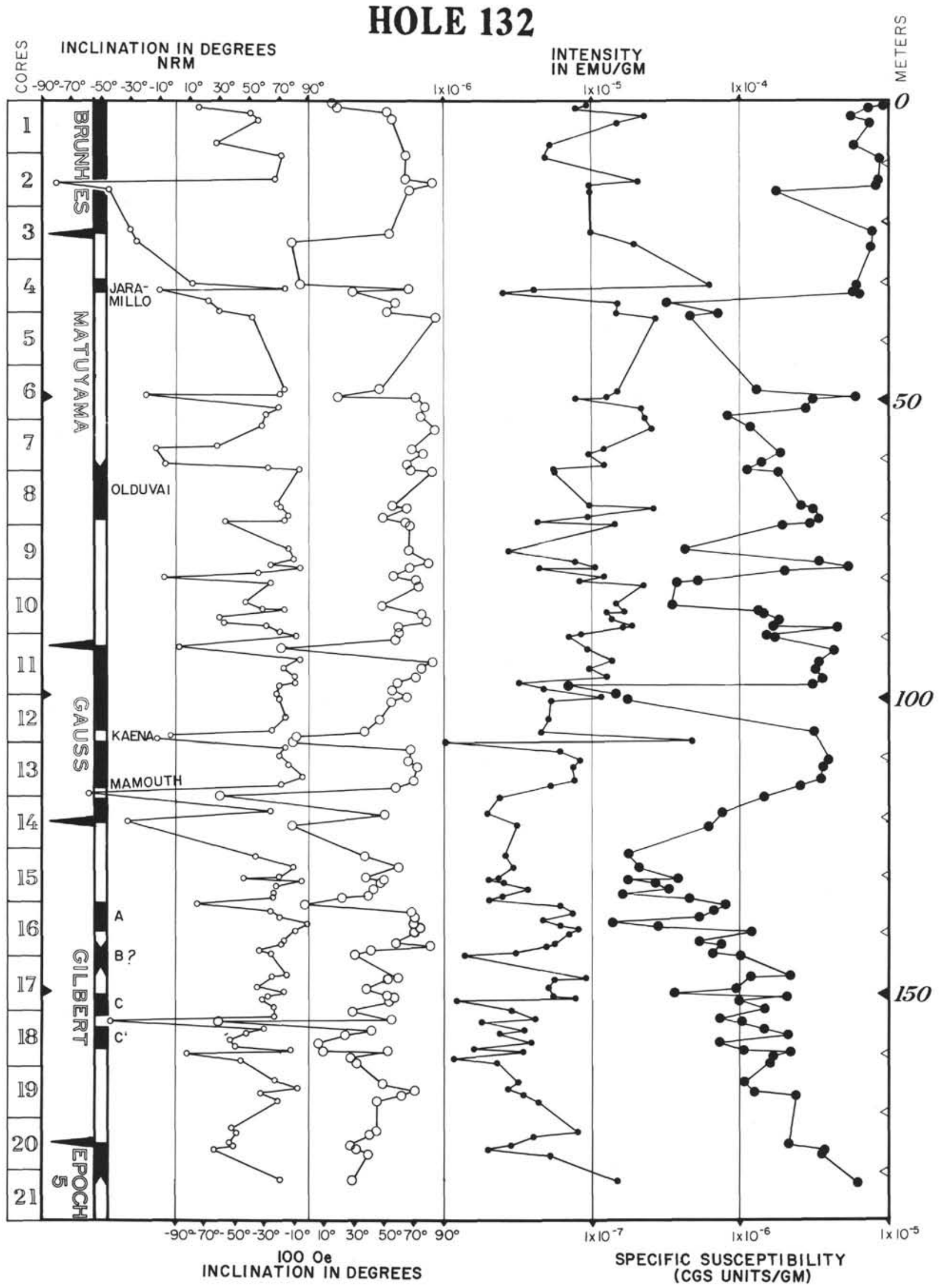

Figure 3. Inclination, intensity and susceptibility profiles for Hole 132. The inclination values are both before demagnetization (NRM) and after cleaning in a field of 100 oersted. The solid bars represent the preliminary interpretation of normal polarity periods. 